\title{
Single Particle versus Collective Electronic Excitations ${ }^{1}$
}

\author{
Philip B. Allen \\ Department of Physics, SUNY, Stony Brook, NY 11794, USA
}

\begin{abstract}
As a first approximation, a metal can be modelled as an electron gas. A non-interacting electron gas has a continuous spectrum of electron-hole pair excitations. At each wavevector $\vec{Q}$ with $|\vec{Q}|$ less than the maximum Fermi surface spanning vector $\left(2 k_{F}\right)$ there is a continuous set of electron-hole pair states, with a maximum energy but no gap (the minimum energy is zero.) Once the Coulomb interaction is taken into account, a new collective mode, the plasmon, is built from the electron-hole pair spectrum. The plasmon captures most of the spectral weight in the scattering cross-section, yet the particle-hole pairs remain practically unchanged, as can be seen from the success of the Landau Fermi-liquid picture. This article explores how even an isolated electron-hole pair in non-interacting approximation is a form of charge density wave excitation, and how the Coulomb interaction totally alters the charge properties, without affecting many other properties of the electron-hole pairs.
\end{abstract}

\section{Introduction}

The low-lying excitations of a non-interacting electron gas are simple rearrangements of the occupancy of the single electron plane-wave orbitals. Because real metals, according to Landau theory, have a lot in common with non-interacting quantum electron gases, this subject is well known to all who study solids. If we neglect band structure, the electron orbitals (labeled by quantum numbers $k=(\vec{k}, \sigma))$ have energy $\epsilon_{k}=\hbar^{2} k^{2} / 2 m$. The ground state has all orbitals occupied which lie inside the Fermi surface, with wavevectors obeying $|\vec{k}|<k_{F}$ and energies obeying $\epsilon_{k}<\epsilon_{F}$, where $k_{F}$ and $\epsilon_{F}$ are the Fermi wavevector and energy. The simplest excitation, known as an electron-hole (e-h) pair, consists of moving an electron out of a state $\vec{k}$ below the Fermi surface, putting it into a state $\vec{k}+\vec{Q}$ above. This is shown in Fig. (1).

The Coulomb interaction is numerically not small, but Landau theory argues that nevertheless, the consequences of the Coulomb interaction are less drastic than one might suppose. However, one drastic effect certainly happens, namely, out of the e-h pair excitation spectrum, the Coulomb interaction creates a new, collective excitation, the plasmon. This typically lies at quite a high energy. Fig. (2) shows the spectrum predicted in random phase approximation (RPA) for sodium metal. The plasmon has an energy $\hbar \omega_{P}(Q)$ which starts at $\approx 6$ eV at $\vec{Q}=0$ and disperses upwards in energy. Also shown in this picture is the continuous spectrum of e-h pairs. This spectrum is easily understood from Fig. (1). At any fixed value of $\vec{Q}$ with $|\vec{Q}|<2 k_{F}$, it is possible to find orbitals $\vec{k}$ just below the Fermi surface such that the corresponding orbital

\footnotetext{
${ }^{1}$ This article was published in the book From Quantum Mechanics to Technology, edited by Z. Petru, J. Przystawa, and K. Rapcewicz (Springer, Berlin, 1996) pp. 125-141.
} 


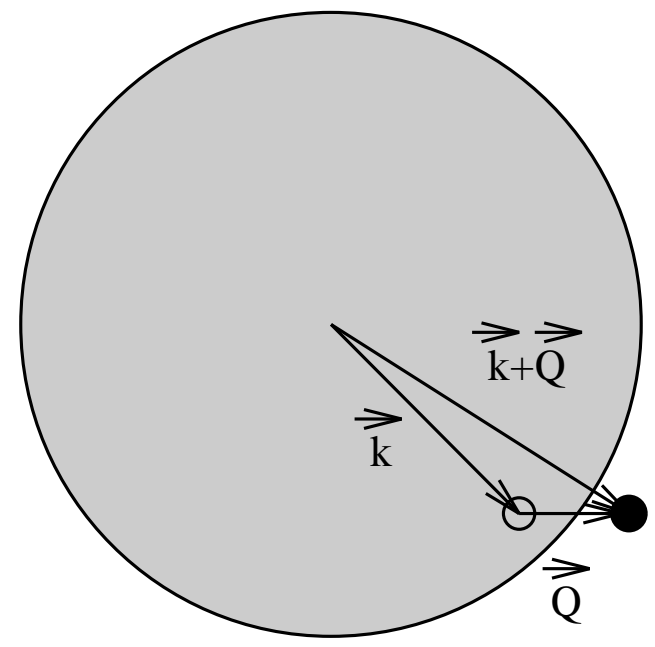

Figure 1: An electron-hole pair excitation, denoted $\mid \vec{k}, \vec{k}+\vec{Q}>$, in a non-interacting electron gas.

$\vec{k}+\vec{Q}$ lies just above the Fermi surface. This means that pair excitations exist with arbitrarily small excitation energies for $Q<2 k_{F}$, whereas for larger $Q$, a gap exists to the lowest single pair excitation. For any $\vec{Q}$ there is also a maximum energy e-h pair which can be created, namely when the hole state $\vec{k}$ lies just below the Fermi surface in the direction of $\vec{Q}$, and the corresponding electron state then lies as far as possible outside the Fermi sea. This excitation has energy $\hbar^{2}\left(k_{F}+Q\right)^{2} / 2 m-\hbar^{2} k_{F}^{2} / 2 m$. This formula gives the upper edge of the e-h pair continuum shown in Fig. (2). Surprisingly, in spite of the totally new plasmon found in RPA, nevertheless, the spectrum of e-h pairs is not altered from the free electron value in RPA.

This subject has been understood for more than 30 years. Nevertheless, the standard treatments in solid state and most many-body texts [1] do not discuss certain aspects which I find paradoxical. This article is intended as pedagogical, aiming to state and then to explain these paradoxes as clearly as possible. Of course, there is no actual paradox in the existing theory, which provides successful approximate methods for calculating many properties of metals, but the most popular approaches use language in which these interesting paradoxical issues are never apparent.

The paradoxes are forcefully apparent in two very interesting experimental studies of Raman scattering by electronic excitations, by Contreras, Sood, and Cardona [2]. The opening paragraph of the first of these papers reads:

"Metals and heavily doped (degenerate) semiconductors can scatter light either through single-particle or collective excitations of free carriers. The single-particle excitations correspond to charge-density fluctuations and, as such, they are screened at low frequencies in a selfconsistent manner by the electrons themselves. Thus, in simple, freeelectron-like carrier systems, no low-frequency scattering is observed. Instead, a peak at the plasma frequency is seen."

The "single-particle" excitations referred to above are just the e-h pair excitations. How close is 


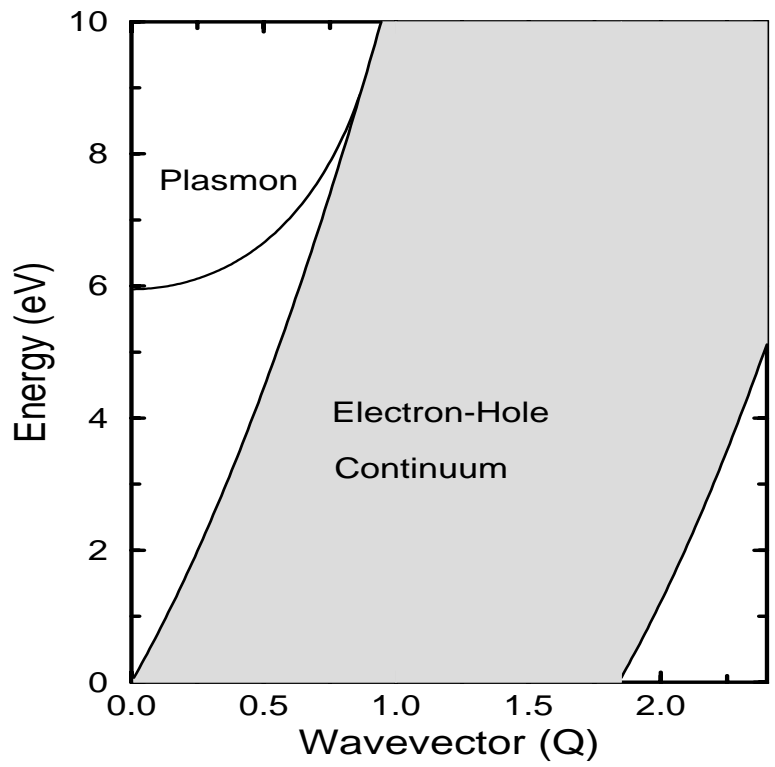

Figure 2: The plasmon dispersion curve and the e-h pair spectrum as calculated in RPA using parameters appropriate to metallic sodium in free electron approximation. The wavevector is in units of $\AA^{-1}$.

this "correspondence" of e-h pairs to charge-density fluctuations? In my own case, the closeness was hard to appreciate at first. After all, the charge density difference between an excited e-h pair state and the ground state (elaborated later) must be just $\delta \rho=\left|\psi_{k+Q}\right|^{2}-\left|\psi_{k}\right|^{2}$, which is zero for plane-wave states. I shall show later that the correspondence is actually perfect. To nuclear physicists this correspondence is quite familiar. Referring to excitations of the nucleus, Brown [3] says

"... we wish to talk about vibrations, which are density fluctuations or

- in quantum-mechanical language - particle-hole excitations ..."

To summarize, the apparent paradoxes are these:

1. How can an e-h pair excitation be equivalent to a charge-density fluctuation?

2. If screening (by the Coulomb interaction) eliminates nearly all the low-frequency scattering of light in favor of collective plasma oscillations, how can it be that the low-lying (e-h pair) spectrum remains unaltered in other experiments (specific heat, susceptibility, conductivity) and in Landau theory?

\section{History and Standard Interpretation}

Electron density oscillations were suspected in electrical discharges in gases, and this subject was elucidated, both experimentally and theoretically, by Tonks and Langmuir [4]. Apparently the corresponding effect for electrons in metals was seen experimentally before being understood 
theoretically. Experiments by Ruthemann [5] and Lang [6] stimulated Kronig, Korringa, and Kramers to recognize the connection to the classical plasma oscillations seen by Tonks and Langmuir. Slater [7] has summarized the history. Bohm and Pines then wrote a series of papers which recognized the importance of the collective plasma degrees of freedom for understanding the interacting electron gas problem. In a review article, Pines [8] coined the term "plasmon", and ever since, the solid state texts have recognized the plasmon as one of the elementary excitations, or quasiparticles, of solid state physics.

The standard derivation of the frequency of plasma oscillations, appearing in all the texts, is identical to an argument from Tonks and Langmuir [4]. Consider a slab of thickness $D$ and infinite transverse size, embedded in an infinite sample of metal with electron density $n$. Imagine that the electrons in this slab are all displaced by the same small amount $u$ in the direction normal to the slab (call this the "upward" direction.) Then a thin layer of charge of thickness $u$ and surface charge density $\sigma=-n e u$ accumulates at the upper surface of the slab and $+n e u$ at the lower surface. Therefore there is a capacitor-type $E$-field of magnitude $4 \pi \sigma=4 \pi n e u$ in the upward direction inside the slab. This field exerts a force $-e E$ in the downward direction on every electron in the slab. This is a restoring force $-K u$ proportional to the displacement of each electron. This causes each electron in the slab to oscillate at frequency $\sqrt{ }(K / m)$, namely,

$$
\omega_{P}^{2}=4 \pi n e^{2} / m
$$

The plasmons in metals are quantized versions of these classical oscillators, with energy $\hbar \omega_{P}$.

The other standard textbook result is that the frequency of plasma oscillations is best understood or calculated by looking for the zero of the real part of the complex dielectric function $\epsilon_{1}(Q, \omega)+i \epsilon_{2}(Q, \omega)$. Even better, the Fourier transform $S(Q, \omega)$ of the density-density correlation function $\left\langle\rho(r, t) \rho\left(r^{\prime}, 0\right)>\right.$, gives the spectrum of density oscillations in a material. Van Hove showed that in Born approximation, the inelastic scattering cross section for particles (like X-rays and electrons) which couple to electron density is given by $S(Q, \omega)$, which is also called the inelastic structure factor. Finally, the same function, $S(Q, \omega)$, is directly proportional to $\operatorname{Im}(-1 / \epsilon(Q, \omega))=\epsilon_{2} /\left(\epsilon_{1}^{2}+\epsilon_{2}^{2}\right)$. Thus the zeros of $\epsilon_{1}(Q, \omega)$ correspond to peaks in $S(Q, \omega)$ and to peaks in the inelastic scattering cross section, provided the damping, given by $\epsilon_{2}(Q, \omega)$ is small. When $\epsilon(Q, \omega)$ is calculated in RPA, the zeros of $\epsilon_{1}$ give the plasmon dispersion shown in Fig. (2). The boundaries of the e-h continuum coincide with the region of $(Q, \omega)$ where $\epsilon_{2}$ differs from zero. In that region, plasmons are very heavily damped, and merge smoothly into the e-h pair spectrum.

The best test of the ideas of collective plasmon excitations is experiment. In the past, inelastic electron scattering away from $\vec{Q}=0$ and also inelastic x-ray scattering with energy resolution better than $1 \mathrm{eV}$ have both been difficult. Recently, synchrotron $\mathrm{x}$-ray sources have made the latter experiment much easier, and we can expect many new results on collective electron behavior [9]. A good example is the measured dispersion curve of plasmons in $\mathrm{Na}$, as determined by inelastic electron scattering by vom Felde et al. [10] and shown in Fig. (3) These results demonstrate that at least in certain simple metals, sharp plasmons exist, and that the RPA is at least qualitatively very successful in explaining the spectrum.

\section{Charge density of an Electron-Hole Pair}




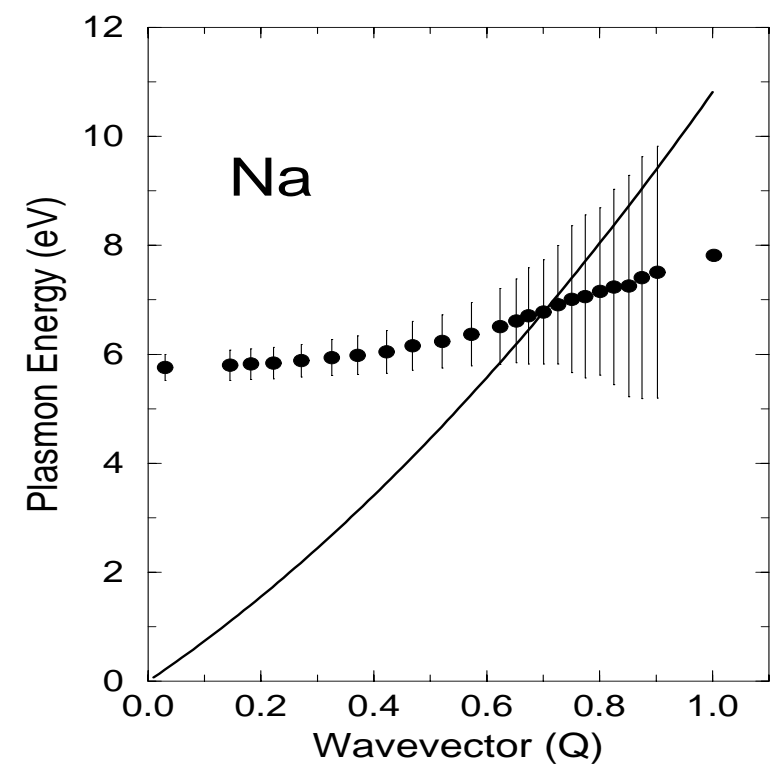

Figure 3: Plasmon dispersion for sodium measured in Ref. [10]. The vertical bars are the measured widths of the plasmon resonances. $Q$ is measured in units $A^{-1}$. The solid curve marks the edge of the e-h pair spectrum as given in Fig. (2). The datum at the largest $Q$ had such a broad lineshape that no sensible width could be assigned.

Contreras et al [2] state that an e-h pair is a charge-density wave excitation. The simple truth of this statement does not emerge in any of the textbook treatments I know. Let us establish some notation. In a non-interacting gas, the ground state can be written as

$$
\left|0>=\prod_{k}^{\text {occ }} c_{k}^{\dagger}\right| \operatorname{vac}>.
$$

Here I am using the notation $k$ as a shorthand for all the quantum numbers of the orbital, that is, the wavevector $\vec{k}$ as well as the spin quantum number and (in a real material) the band index. An e-h pair excitation is denoted by

$$
\left|k, k+Q>=c_{k+Q}^{\dagger} c_{k}\right| 0>
$$

This state vanishes unless the orbital $k$ is occupied in the ground state, and the orbital $k+Q$ is empty.

The charge density is $-e$ times the electron density. I will leave out the factor $-e$ and refer to it as charge density anyway. To find the charge density of the e-h pair state, one would calculate the expectation value of the charge density operator,

$$
\hat{\rho}(\vec{r}, t)=\hat{\psi}^{\dagger}(\vec{r}, t) \hat{\psi}(\vec{r}, t)
$$

where the field operator $\hat{\psi}$ can be represented in terms of the one electron states as

$$
\hat{\psi}(\vec{r}, t)=\sum_{k} \psi_{k}(\vec{r}) c_{k}(t)
$$


In a one-electron approximation, or else in the "interaction representation", $c_{k}(t)$ has the form $\exp \left(-i \epsilon_{k} t / \hbar\right) c_{k}$. In the ground state, the particle density is

$$
\rho_{0}(\vec{r}, t)=<0|\hat{\rho}(\vec{r}, t)| 0>=<0\left|\sum_{p, p^{\prime}} \psi_{p}^{*}(\vec{r}) \psi_{p^{\prime}}(\vec{r}) c_{p}^{\dagger}(t) c_{p^{\prime}}(t)\right| 0>
$$

Only the diagonal terms $p=p^{\prime}$ which are occupied give a non-zero contribution to this sum, and we get the familiar result, independent of time,

$$
\rho_{0}(\vec{r})=\sum_{p}^{\mathrm{occ}}\left|\psi_{p}(\vec{r})\right|^{2}
$$

Repeating the calculation for the e-h pair state $|k, k+Q\rangle$, we get the same answer except that the list of occupied states is different. If we look at the change in the charge density between the ground state and the e-h pair state, we get

$$
\delta \rho(\vec{r}, t)=\left|\psi_{k+Q}(\vec{r})\right|^{2}-\left|\psi_{k}(\vec{r})\right|^{2} .
$$

For free electrons, the orbitals $\psi_{k}(\vec{r})$ are plane waves $\exp (i \vec{k} \cdot \vec{r})$, and $\delta \rho$ vanishes. For the Bloch states of a real metal, the orbitals are plane waves modulated by periodic functions $u_{k}(\vec{r})$. For small $Q$, the periodic function $u_{k+Q}$ is almost the same as $u_{k}$, so the change in charge density vanishes as $Q$ goes to zero. There is no evidence of a charge-density wave.

Nevertheless, Contreras et al. [2] are right. There is a charge-density wave hidden in the pair state. The way to see it is to consider what would happen if a small amount of e-h pair excitation were mixed with the ground state. In other words, consider the state

$$
\left|\Psi_{k, k+Q}>\equiv\right| 0>+\eta\left|k, k+Q>=\left(1+\eta c_{k+q}^{\dagger} c_{k}\right)\right| 0>
$$

where $\eta$ is a small complex number, and it is assumed that the pair excitation does not vanish, i. e. that $k$ is inside the Fermi distribution and $k+Q$ outside. The calculation now yields (to first order in the small parameter $\eta$ )

$$
\delta \rho=\eta \psi_{k+Q}^{*}(\vec{r}) \psi_{k}(\vec{r}) e^{-i\left(\epsilon_{k+Q}-\epsilon_{k}\right) t / \hbar}+\text { c.c. }
$$

where c.c. stands for "complex conjugate." For small $\vec{Q}$, the electron-hole pair energy $\left(\epsilon_{k+Q}-\right.$ $\left.\epsilon_{k}\right) / \hbar$ is $\vec{Q} \cdot \vec{v}_{k}$ where $\vec{v}_{k}$ is the group velocity, $\partial \epsilon_{k} / \partial \vec{k}$. At the same level in smallness of $\vec{Q}$, we can write the product of wavefunctions as

$$
\psi_{k+Q}^{*}(\vec{r}) \psi_{k}(\vec{r})=\left|\psi_{k}(\vec{r})\right|^{2} e^{i \vec{Q} \cdot \vec{r}}
$$

This is just the zeroth order result of a $" \vec{k} \cdot \vec{p} "$ perturbation theory. Substituting these changes into Eq. (10), we get the result

$$
\delta \rho(\vec{r}, t)=2|\eta|\left|\psi_{k}(\vec{r})\right|^{2} \cos \left[\vec{Q} \cdot\left(\vec{r}-\vec{v}_{k} t\right)+\phi\right]
$$

where $\phi$ is the phase of $\eta$. Now we see that the e-h pair excitation, relative to the ground state, is a charge-density wave! The propagation direction is of course given by $\vec{Q}$, and the phase 
velocity is the component $\hat{Q} \cdot \vec{v}_{k}$ of the electron group velocity in the direction of $\vec{Q}$. The group velocity, however, is $\vec{v}_{k}$. The simple cosine oscillation of electron density is very reminiscent of a phonon. However, unlike phonons which have 3 branches for each atom in the cell, the number of e-h pairs at a fixed $\vec{Q}$ is a macroscopic number. Specifically, for free electrons with $Q>2 k_{F}$, the number of pairs with wavevector $\vec{Q}$ is the same as the number of occupied electron states, whereas for $Q<2 k_{F}$, the number is reduced by a factor $(3 / 2)\left(Q / 2 k_{F}\right)\left[1-\left(Q / 2 k_{F}\right)^{2} / 3\right]$.

It is evident from the electrostatic argument of Tonks and Langmuir that once the Coulomb interaction is taken into account, there will be a large additional restoring force on the charge oscillation, and the result will be that the charge density will oscillate at the plasma frequency rather than the non-interacting frequency $\vec{Q} \cdot \vec{v}_{k}$. This will be shown explicitly in the next section. One should pause to ask what property of the medium permits wave propagation (Eqn. (12)) of electron charge before the Coulomb interaction is turned on. If there is no interaction, a classical gas does not support propagating waves. The answer is the Fermi degeneracy, which forces an energy cost if the local density is altered.

\section{Influence of Coulomb Interaction on Charge of the e-h Pair}

It is convenient to formulate this as a linear response problem. This way we expect the Coulomb interaction to enter nicely as a dielectric screening. Therefore, we want a perturbing field which will create the e-h pair and which can be represented as a term in the Hamiltonian. The following operator does the trick:

$$
H^{\prime}=\hbar \eta c_{k+Q}^{\dagger} c_{k} \delta(t)
$$

This inserts an electron-hole pair at time zero. The dimensionless strength of the insertion field, $\eta$, will be taken to be a small number. Linear response theory provides formal answers for the alterations of system properities which can be measured at a later time, to first order in $\eta$. In this section, we explore the resulting charge-density response, that is, the expectation value of the operator $\hat{\rho}$ of Eqn. (4). Later we look at the current response.

Standard techniques of linear response theory [11] tell us that the response is

$$
\delta \rho(\vec{r}, t)=\frac{1}{i \hbar} \int_{-\infty}^{t} d t^{\prime}\left\langle 0\left|\hat{\rho}(t) H^{\prime}\left(t^{\prime}\right)-H^{\prime \dagger}\left(t^{\prime}\right) \hat{\rho}(t)\right| 0\right\rangle .
$$

This is a standard Kubo-type formula, except that since the Hamiltonian $H^{\prime}$ is not Hermitean, so the commutator is replaced by a slighly more complicated form. Inserting Eqn. (13) for $H^{\prime}$, the charge density response is

$$
\begin{gathered}
\delta \rho(\vec{r}, t)=2 \operatorname{Im}\{\eta D(t)\} \theta(t) \\
D(t)=<0\left|\hat{T} \hat{\rho}(\vec{r}, t) c_{k+q}^{\dagger} c_{k}\right| 0>
\end{gathered}
$$

where $\theta(t)$ is 1 if $t>0$ and zero otherwise. $D$ is a type of two-particle Green's function, and can be evaluated perturbatively in powers of the Coulomb interaction by standard Feynman methods using time-ordered Green's functions. The time-ordering operator $\hat{T}$ has been inserted 
into Eqn. (16). Since ultimately we only need to know $D$ for positive times, this doesn't change anything but facilitates the perturbation theory.

First, evaluate the charge response function without any Coulomb interactions, $D_{0}$. By standard methods one gets

$$
\begin{gathered}
D_{0}(t)=e^{i \vec{Q} \cdot \vec{r}} \int_{-\infty}^{\infty} \frac{d \omega}{2 \pi} e^{-i \omega t} D_{0}(\omega) \\
D_{0}(\omega)=i\left\{\frac{f_{k}\left(1-f_{k+Q}\right)}{\omega+i \eta-\vec{Q} \cdot \vec{v}_{k}}-\frac{f_{k+Q}\left(1-f_{k}\right)}{\omega-i \eta-\vec{Q} \cdot \vec{v}_{k}}\right\}
\end{gathered}
$$

For positive $t$, the contour is closed in the lower complex $\omega$ plane, and only the first term in $D_{0}(\omega)$ contributes. The answer is

$$
\delta \rho(\vec{r}, t)=2|\eta| \sin \left(\vec{Q} \cdot \vec{r}-\vec{Q} \cdot \vec{v}_{k} t+\phi\right)\left|\psi_{k}(\vec{r})\right|^{2} f_{k}\left(1-f_{k+Q}\right)
$$

This is just the same as the previous result for the charge density change which occurs when an e-h pair is present with amplitude $\eta$ at all times, Eqn. (12), except for a phase change of $\pi / 2$. This is because our earlier pair oscillator started at time zero with given amplitude $\eta$ whereas the present oscillator at time zero was given "velocity" by the impulsive insertion. The present version also contains explicitly the Fermi factors $f_{k}$ and $\left(1-f_{k+Q}\right)$ which are zero or one depending on whether the orbital is within or without the Fermi distribution.

The Coulomb interaction has the form

$$
\hat{V}=\frac{1}{2} \sum_{Q^{\prime}} \hat{\rho}_{-Q^{\prime}} v\left(Q^{\prime}\right) \hat{\rho}_{Q^{\prime}}
$$

where the operator $\hat{\rho}(Q)$ is the Fourier transform of the charge density operator Eqn. (4). For free electrons this is just

$$
\hat{\rho}_{Q}=\sum_{k} c_{k+Q}^{\dagger} c_{k}
$$

To evaluate the response function $D$ exactly in the presence of Coulomb interactions is of course impossible. Diagrammatic perturbation theory allows a classification of the correction terms. The RPA is a standard approximation which keeps an infinite set of leading diagrams (which contain the leading small $\vec{Q}$ divergence, or the long-range part of the Coulomb interaction). The result at RPA level is

$$
D_{R P A}(t)=e^{i \vec{Q} \cdot \vec{r}} \int_{-\infty}^{\infty} \frac{d \omega}{2 \pi} \frac{D_{0}(\omega) e^{-i \omega t}}{1-v(Q) \chi_{0 T}(\vec{Q}, \omega)}
$$

where $\chi_{0}$ is the usual non-interacting susceptibility "bubble" diagram, and the subscript $T$ indicates that it is the "time-ordered" rather than the usual "retarded" or causal response function $\chi_{0 R}$ that is needed. The factor $v(Q)$ is $4 \pi e^{2} / Q^{2}$, and the denominator $1-v(Q) \chi_{0}$ is the dielectric function $\epsilon(Q, \omega)$. These formulas are written for the uniform electron gas with no background of actual atoms. Generalizing to real electrons interacting with a crystalline array of atoms is not hard, but complicates the notation because of the matrix nature of $\epsilon$. At this 
stage it is also easy to include the dominant impurity effects, simply by including them in $\epsilon$. If the mean free path $\ell=v_{F} \tau$ is short enough that $Q \ell \ll 1$, the standard Drude result applies,

$$
\epsilon_{R}(Q, \omega)=1-\frac{\omega_{p}^{2}}{\omega(\omega+i / \tau)}
$$

It is important to convert this to the retarded form, which can be done using the general relations

$$
\begin{gathered}
\operatorname{Re} \chi_{R}(\omega)=\operatorname{Re} \chi_{T}(\omega) \\
\operatorname{Im} \chi_{R}(\omega)=\tanh \left(\frac{\hbar \omega}{2 k_{B} T}\right) \operatorname{Im} \chi_{T}(\omega)
\end{gathered}
$$

At low $T$, the hyperbolic tangent is $\approx \pm 1$ and to convert a retarded function to a time-ordered function, one just changes the sign of the imaginary part when $\omega<0$. To a good approximation the Drude response function can be written

$$
\frac{1}{\epsilon_{R}} \cong \frac{1}{2 \omega_{P}}\left[\frac{\omega+i / \tau}{\omega-\omega_{P}+i / 2 \tau}-\frac{\omega+i / \tau}{\omega+\omega_{P}+i / 2 \tau}\right]
$$

Because $\omega_{P} \gg 1 / \tau$, the first term of Eqn. (26) is only important when $\omega>0$ and the second term is only important when $\omega<0$. Therefore this is converted into a time-ordered response function by the simple approximate expedient of taking the complex conjugate of the second term,

$$
\frac{1}{\epsilon_{T}}=\frac{\omega}{2 \omega_{p}}\left[\frac{\omega+i / \tau}{\omega-\omega_{p}+i / 2 \tau}-\frac{\omega-i / \tau}{\omega+\omega_{p}-i / 2 \tau}\right]
$$

Putting this into Eqn. (22) and closing the contour in the lower half of the complex $\omega$ plane, the result is

$$
\begin{aligned}
D_{R P A}(t) & =e^{i\left(\vec{Q} \cdot \vec{r}-\omega_{p} t\right)} e^{-t / 2 \tau}\left(f_{k}-f_{k+Q}\right) / 2 \\
& +e^{i\left(\vec{Q} \cdot \vec{r}-\vec{Q} \cdot \vec{v}_{k} t\right)} f_{k}\left(1-f_{k+Q}\right) \times \mathcal{O}\left(\frac{Q v_{F} \text { or } 1 / \tau}{\omega_{p}}\right)^{2}
\end{aligned}
$$

For simplicity, the Bloch wavefunction $\left|\psi_{k}\right|^{2}$ has been dropped. Only free electron results are given for the remainder of this section. The second term of Eqn. (28) is negligible compared to the first at small $Q$. Therefore, using Eqn. (15), the result for the charge disturbance after accounting for the long range Coulomb field is

$$
\delta \rho(\vec{r}, t)=|\eta| \sin \left(\vec{Q} \cdot \vec{r}-\omega_{p} t\right) e^{-t / 2 \tau}\left(f_{k}-f_{k+Q}\right) .
$$

This is almost what one would have naively guessed. The amplitude is reduced by 2 , and the frequency is now the plasma frequency, $\omega_{p}$, as expected, with only a negligible component left oscillating at the original frequency $\vec{Q} \cdot \vec{v}_{k}$. Compensating for what looks like an arbitrary reduction by half of the amplitude is a new effect, coming from the difference between the factors $f_{k}\left(1-f_{k+Q}\right)$ and $\left(f_{k}-f_{k+Q}\right)$. In the interacting system, it is possible to insert an 


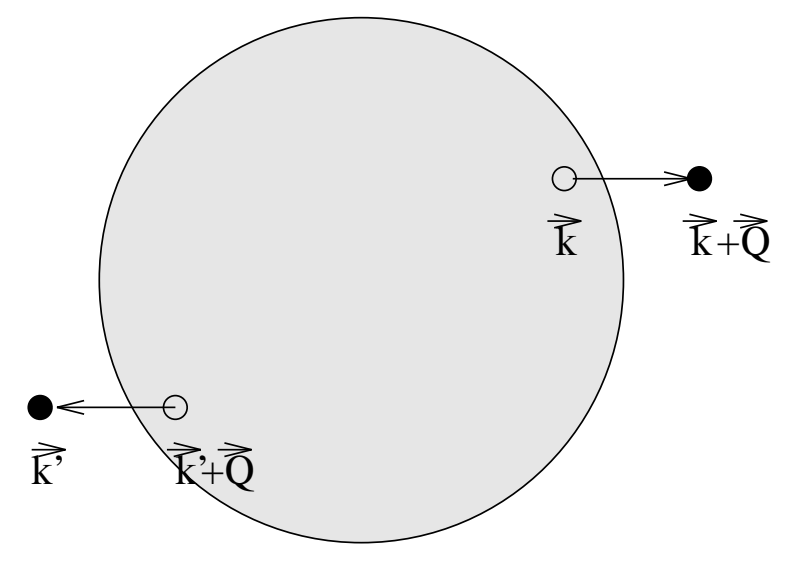

Figure 4: A typical vacuum fluctuation, consisting of two e-h pairs with momentum $\vec{Q}$ and $-\vec{Q}$.

electron-hole pair even when the state $k$ is above the Fermi surface $\left(f_{k}=0\right)$ and the state $k+Q$ is below $\left(f_{k+Q}=1\right)$. The factor $f_{k}\left(1-f_{k+Q}\right)$ of the non-interacting response is zero, but the factor $\left(f_{k}-f_{k+Q}\right)$ of the interacting system is -1 (the density disturbance has a phase shift of $\pi$.) Where does this new term come from? The Coulomb interaction Eqn. (20) continuously creates "vaccuum fluctuations" which are pairs of electron-hole pairs of equal and opposite momentum $Q^{\prime}$ and $-Q^{\prime}$, as shown in Fig. 4. Thus there is the possibility that an electron with $k>k_{F}$ and a hole with $k+Q<k_{F}$ are already present at time zero because of a vaccuum fluctuation (which also created a pair $k^{\prime}<k_{F}$ and $k^{\prime}+Q>k_{F}$ ). Then the "insertion" operator $c_{k+Q}^{\dagger} c_{k}$ removes the already present electron-hole pair, leaving its mate $k^{\prime}, k^{\prime}+Q$. This process has equal amplitude as the simpler insertion process, but each is reduced by 2 compared to the non-interacting case.

\section{Current Density of an Electron-Hole Pair in a Metal}

It is also interesting to ask what is the current density associated with an electron-hole pair excitation. The current density operator, analogous to Eqn. (4), is

$$
\hat{\vec{j}}(\vec{r})=\frac{\hbar}{2 m i}\left[\hat{\psi}^{\dagger}(\vec{r})(\vec{\nabla} \hat{\psi}(\vec{r}))-(\vec{\nabla} \hat{\psi}(\vec{r}))^{\dagger} \hat{\psi}(\vec{r})\right]
$$

The ground state carries no current. To first order in the amplitude $\eta$, the current density in the state $\mid \Psi_{k, k+Q}>($ Eqn. 9$)$ is

$$
\delta \vec{j}(\vec{r})=\frac{\hbar}{2 m i} \eta\left[\psi_{k}^{*}(\vec{r})\left(\vec{\nabla} \psi_{k+Q}(\vec{r})\right)-\left(\vec{\nabla} \psi_{k}^{*}(\vec{r})\right) \psi_{k+Q}(\vec{r})\right] e^{-i\left(\epsilon_{k+Q}-\epsilon_{k}\right) t / \hbar}+\text { c.c. }
$$

Because the wavevector $\vec{Q}$ is small, the factor inside the square brackets of Eqn. (31) can be written as

$$
\frac{\hbar}{2 m i}[\quad]=e^{i \vec{Q} \cdot \vec{r}} \vec{j}_{k}(\vec{r})
$$




$$
\vec{j}_{k}(\vec{r})=\frac{\hbar}{2 m i}\left[\psi_{k}^{*}\left(\vec{\nabla} \psi_{k}\right)-\left(\vec{\nabla} \psi_{k}^{*}\right) \psi_{k}\right]
$$

where $\vec{j}_{k}(\vec{r})$ is the current density associated with the single particle Bloch state $\psi_{k}(\vec{r})$. Since the corresponding density $\left|\psi_{k}(\vec{r})\right|^{2}$ is time-independent, the vector field $\vec{j}_{k}(\vec{r})$ must be divergenceless. The spatial average of this current density is $\vec{v}_{k} / \Omega$ where $\vec{v}_{k}$ is the group velocity of the state and $\Omega$ is the volume of the crystal. Thus we get

$$
\delta \vec{j}(\vec{r})=2|\eta| \vec{j}_{k}(\vec{r}) \cos \left[\vec{Q} \cdot\left(\vec{r}-\vec{v}_{k} t\right)+\phi\right] f_{k}\left(1-f_{k+Q}\right)
$$

The electron-hole pair conserves charge while it propagates, that is $\vec{\nabla} \cdot \vec{j}+\partial \rho / \partial t$ is zero. However, Eqs. (12) and (34) do not rigorously obey this law, since the current $\vec{j}_{k}$ is not equal everywhere to $\vec{v}_{k}\left|\psi_{k}\right|^{2}$, only on average. The more exact forms (10) and (31) are rigorously charge-conserving. The derivatives $\vec{\nabla} \cdot \vec{j}$ and $\partial \rho / \partial t$ are first order in $|\vec{Q}|$, whereas $\vec{j}$ and $\rho$ are zeroth order. Since Eqs. (12) and (34) throw away terms first order in $|\vec{Q}|$, they also lose some first order parts of their derivatives, even though they are correct to the order that they are written.

It is interesting that the current density is not in general longitudinal. It is polarized in a direction whose spatial average is the direction of the group velocity $\vec{v}_{k}$, which is normally not parallel to $\vec{Q}$. The transverse part of the current contains new information which cannot be deduced from the density alone.

\section{Influence of Coulomb Interaction on Current of the e-h Pair}

The same linear response proceedure used earlier for the charge can be generalized for the current. Analogous to Eqns. $(15,16)$ we get

$$
\begin{gathered}
\delta \vec{j}(\vec{r}, t)=2 \operatorname{Im}\{\eta \vec{J}(t)\} \theta(t) \\
J_{\alpha}(t)=<0\left|\hat{T} \hat{j}_{\alpha}(\vec{r}, t) c_{k+q}^{\dagger} c_{k}\right| 0>
\end{gathered}
$$

When this is evaluated without Coulomb interactions, the result is

$$
\delta \vec{j}(\vec{r})=2|\eta| \vec{j}_{k}(\vec{r}) \sin \left[\vec{Q} \cdot\left(\vec{r}-\vec{v}_{k} t\right)+\phi\right] f_{k}\left(1-f_{k+Q}\right),
$$

analogous to Eqn. (19).

When Coulomb interactions are accounted for by perturbation theory, we discover that transverse and longitudinal parts of the current are treated very differently. Sums over $\vec{k}^{\prime}$ occur in which there are factors of the type

$$
\left(\vec{v}_{k^{\prime} \perp}+\vec{v}_{k^{\prime} \mid}\right) \times F\left(\epsilon_{k^{\prime}}, \epsilon_{k^{\prime}+Q}\right) .
$$

The velocity $\vec{v}_{k^{\prime}}$ has been split into a transverse part (perpendicular to $\vec{Q}$ ) and a longitudinal part. The transverse contribution vanishes, because it is always possible, for free electrons or for band electrons as long as $\vec{Q}$ has high enough symmetry, to find two states $k^{\prime}$ which 
have identical values of $\epsilon_{k^{\prime}}, \epsilon_{k^{\prime}+Q}$ and $v_{k^{\prime} \|}$ and opposite values of $v_{k^{\prime}}$. Therefore, all Coulomb corrections to the transverse current cancel, and the transverse current continues to oscillate at its unrenormalized frequency.

The longitudinal part of the current gets screened in RPA the same way the charge does. A diagrammatic analysis yields the result

$$
\vec{Q} \cdot \vec{J}(t)=e^{i \vec{Q} \cdot \vec{r}} \int \frac{d \omega}{2 \pi} e^{-i \omega t} \frac{\omega D_{0}(\omega)}{1-v(Q) \chi_{0 T}(\vec{Q}, \omega)}
$$

The final result for the current is

$$
\begin{aligned}
\delta \vec{j}(\vec{r}, t) & =\frac{2|\eta|}{V} \vec{v}_{k \perp} \sin \left(\vec{Q} \cdot \vec{r}-\vec{Q} \cdot \vec{v}_{k} t+\phi\right) f_{k}\left(1-f_{k+Q}\right) \\
& +\frac{|\eta| \omega_{P}}{V Q} \hat{Q} \sin \left(\vec{Q} \cdot \vec{r}-\omega_{P} t+\phi\right) e^{-t / 2 \tau}\left(f_{k}-f_{k+Q}\right)
\end{aligned}
$$

This answer was calculated using the Drude response in the same way that Eqn. (29) was calculated.

We have now learned something interesting about e-h pair excitations. Even though the Coulomb interaction totally alters the charge oscillations of every e-h pair, other properties can remain completely immune to alteration, such as the transverse part of the current oscillation. This is part of the explanation of the second "apparent" paradox. A more complete explanation is given in the next section.

\section{What is the Wavefunction of a Plasmon?}

Another way of understanding the broad immunity of e-h pairs to Coulombic alteration is to recognize that it is easy to combine e-h pairs so that the charge is hidden. A trivial way is to make a random combination of pairs. Rather than a single coherent cosine of charge oscillation as in Eqn. (12), one now expects an incoherent sum of many cosines with different phases, adding up to a disturbance whose charge is smaller than a coherent sum by a factor $1 / \sqrt{ } N$ where $N$ is the number of e-h pairs combined. Then the Coulomb interaction will still alter the charge oscillation, but this is only a minor aspect of the complete excitation.

A way of picturing this more elegantly is to consider the space of all single e-h pair states. The Hamiltonian can couple through the Coulomb interaction only states of the same $\vec{Q}$. Therefore we restrict attention to states of the form

$$
\left|\Psi>=\sum_{k} A_{k}\right| k, k+Q>
$$

where the sum goes over all $k$ 's such that $k$ is below the Fermi surface and $k+Q$ is above. In this subspace the Hamiltonian matrix is

$$
\hat{H}_{1 \mathrm{e}-\mathrm{h}}=\left(\begin{array}{ccc}
\epsilon_{k_{1}+Q}-\epsilon_{k_{1}}+v(Q) & v(Q) & \cdots \\
v(Q) & \epsilon_{k_{2}+Q}-\epsilon_{k_{2}}+v(Q) & \cdots \\
\vdots & \vdots
\end{array}\right)
$$


The kinetic energy of the pair $(k, k+Q)$ appears on the diagonal, and the Coulomb interaction $v(Q)=4 \pi e^{2} / Q^{2}$ couples each pair to every other pair. The exchange term $-v\left(k-k^{\prime}\right)$ also couples pairs to each other, provided their spins are the same, but this is a smaller and a complicating factor which is omitted in first approximation.

We look for eigenstates of this truncated Hamiltonian. This proceedure is called the TammDancoff approximation by nuclear physicists [1], and is an example of what chemists call a "configuration interaction" calculation. The eigenfunctions obey

$$
\hat{H}_{1 \mathrm{e}-\mathrm{h}}|A>=\lambda| A>
$$

which is equivalent to

$$
\left(\epsilon_{k+Q}-\epsilon_{k}-\lambda\right) A_{k}=-v(Q) \sum_{k^{\prime}} A_{k^{\prime}}
$$

Solving for $A_{k}=$ const $\times v(Q) /\left(\epsilon_{k+Q}-\epsilon_{k}-\lambda\right)$, we compute $\sum_{k} A_{k}$ and find the self-consistency condition

$$
1=-v(Q) \sum_{k} \frac{f_{k}\left(1-f_{k+Q}\right)}{\epsilon_{k+Q}-\epsilon_{k}-\lambda}
$$

This is equivalent to the secular equation $\operatorname{det}\left(\hat{H}_{1 \mathrm{e}-\mathrm{h}}-\lambda \hat{1}\right)=0$.

The nature of this equation can be appreciated by considering the form of the right hand side for a small system with a discrete spectrum of e-h pairs of energy $\epsilon_{k+Q}-\epsilon_{k}$. Between every adjacent two pair energies, a root $\lambda$ of Eqn. (45) is found, but the largest root $\lambda=\hbar \omega_{\mathrm{TD}}$ is split off to higher energy as illustrated in Fig. 5. This root is the collective electronic excitation in Tamm-Dancoff approximation. Its wavefunction is

$$
\left|\Psi>=\sum_{k} \frac{C v(Q)}{\hbar \omega_{\mathrm{TD}}-\left(\epsilon_{k+Q}-\epsilon_{k}\right)}\right| k, k+Q>
$$

Because $\hbar \omega_{\text {TD }}$ lies above the energy of the pair spectrum, all the coefficients in Eqn. (46) are positive. This coherent sum has a large oscillating charge at $t \approx 0$, and looks quite a lot like a plasmon. The other eigenstates of Eqn. (42) are orthogonal to 46, which means that at $t \approx 0$ the charge must largely cancel out. These other states account for the persistence of non-interacting properties in the e-h pair spectrum of the electron gas with Coulomb interactions.

Unfortunately, the Tamm-Dancoff approximation, as is well-known in nuclear physics, does not yield an accurate answer for the collective mode spectrum. The RPA answer, which is apparently surprisingly accurate, is equivalent to a modified secular equation

$$
1=-v(Q) \sum_{k} \frac{f_{k}\left(1-f_{k+Q}\right)-f_{k+Q}\left(1-f_{k}\right)}{\epsilon_{k+Q}-\epsilon_{k}-\lambda}
$$

which is found by setting the real part of the RPA dielectric function to zero. Eqn. (45) is tantalizingly close to Eqn. (47). However, the additional term which occurs in Eqn. (47), having the factor $f_{k+Q}\left(1-f_{k}\right)$ in the numerator, is quite foreign to the Tamm-Dancoff approximation, because it seems to refer to states $\mid k, k+Q>$ where the hole state $k$ is above the Fermi surface and the electron state $k+Q$ is below. 


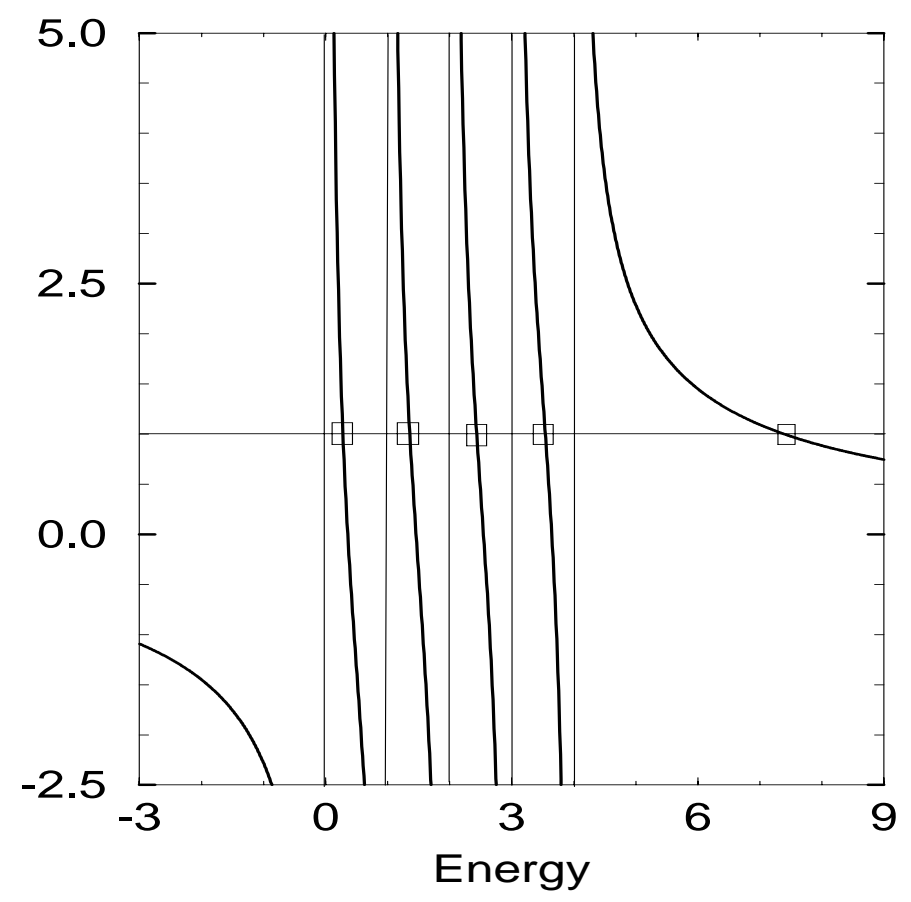

Figure 5: Finding the roots of the secular equation. The curve is the right hand side of Eqn. (45). The vertical lines mark the energies of e-h pair states, with a root pinned between each two neighboring e-h states. One root (which becomes the plasmon) splits off to higher energy.

The pair creation operator $c_{k+Q}^{\dagger} c_{k}$ operating on the non-interacting ground state cannot create anything if $k$ is above the Fermi surface and $k+Q$ below, but if it acts on the interacting ground state with vaccuum fluctuations included, then the pair creation operator can destroy a pair of net momentum $-\vec{Q}$ by destroying a virtual electron in state $k$ and a virtual hole in state $k+Q$. This effectively releases a pair state in some other state $\left(k^{\prime}, k^{\prime}+Q\right)$ as shown in Fig. 4, which had previously been part of the same vaccuum fluctuation as the destroyed pair.

In order to produce the modified secular Eqn. (47) in place of Eqn. (45) from our wavefunction argument, we need an enlarged subspace that includes e-h pairs where the hole is above and the electron below the Fermi level. The kinetic energy part of the Hamiltonian will have the same form $\epsilon_{k+Q}-\epsilon_{k}$ on the diagonal in both the previous and the new parts of the subspace, whereas the potential energy term must have the form

$$
\hat{V}_{e-h}=\left(\begin{array}{ccc|ccc}
v(Q) & v(Q) & \cdots & -v(Q) & -v(Q) & \cdots \\
v(Q) & v(Q) & \cdots & -v(Q) & -v(Q) & \cdots \\
\vdots & \vdots & & \vdots & \vdots & \\
\hline-v(Q) & -v(Q) & \cdots & v(Q) & v(Q) & \cdots \\
-v(Q) & -v(Q) & \cdots & v(Q) & v(Q) & \cdots \\
\vdots & \vdots & & \vdots & \vdots &
\end{array}\right)
$$

where the second set of rows and columns refers to the new states with $k$ above and $k+Q$ below the Fermi level. One way to begin building this is to redefine the pair states given in 
Eqn. (3) by a first-order perturbative improvement of the ground state or vaccuum,

$$
|0>\rightarrow| 0>+\sum_{m} \mid m>\frac{<m|\hat{V}| 0>}{E_{0}-E_{m}}
$$

where $\mid m>$ is short for any state (consisting of two e-h pairs of opposite momenta $\vec{Q}^{\prime},-\vec{Q}^{\prime}$ ) which can be created in the non-interacting vaccuum by the Coulomb interaction Eqn. (20). The original subspace Hamiltonian matrix Eqn. (42) is preserved to zeroth order in $v(Q)$, and to first order, there are new matrix elements of the kinetic energy operator which couple an e-h pair $(k, k+Q)$ with $k$ below the Fermi level to a state with $k^{\prime}$ above. Unfortunately, the coupling matrix element is not just the term $-v(Q)$ appearing in Eqn. (48), but instead

$$
-v(Q) \times \frac{\epsilon_{k+Q}-\epsilon_{k}}{\left(\epsilon_{k+Q}-\epsilon_{k}\right)-\left(\epsilon_{k^{\prime}+Q}-\epsilon_{k^{\prime}}\right)}
$$

It will require going to infinite order in perturbation theory to fully correct the Tamm-Dancoff approximation and recover the RPA answer by this route. The Tamm-Dancoff approximation is equivalent to a diagrammatic perturbation theory for the dielectric function which keeps only one e-h pair propagating forward in time, and neglects the backward-in-time propagation which enters the usual Dyson series when the vaccuum fluctuations are included properly in the same order of perturbation theory. The full RPA treatment can have arbitrarily many additional vaccuum fluctuations with wavevectors $(\vec{Q},-\vec{Q})$, but our approximate improvement allows at most one vaccuum fluctuation term. It might be nice to find explicitly the formula for the plasmon wavefunction which corresponds properly to the RPA formula, but I have not done this. The subject is treated in texts [1], [3] on the nuclear many-body problem.

\section{Conclusion}

The apparent paradoxes now seem largely answered. The answers did not involve any new physics, but instead required thinking about the problem in a way familiar to nuclear physicists but less familiar to solid state physicists. An e-h pair, even before adding the Coulomb interaction, is seen to carry a charge density wave, once the interference between the pair and the ground state is considered in the wavefunction. The Coulomb interaction totally alters the charge-carrying part of this state, but does not affect the transverse part of the current. In the effort to find approximate eigenstates of the interacting problem, the Tamm-Dancoff proceedure, even though ultimately inaccurate, offers a nice way of seeing how a single plasmon-like mode can split off from the e-h continuum, leaving the rest of the continuum largely unaltered.

\section{Acknowledgements}

I thank G. E. Brown, M. Cardona, V. J. Emery, A. D. Jackson, and especially J. K. Jain for helpful discussions. This work was supported in part by NSF grant no. DMR-9417755. I thank Prof. R. Car for hospitality at IRRMA in Lausanne, where the first half of this work was done. 


\section{References}

[1] A particularly complete treatment of the RPA and plasmons is given by A. L. Fetter and J. D. Walecka, Quantum Theory of Many-Particle Systems (McGraw-Hill, New York, 1971).

[2] G. Contreras, A. K. Sood, and M. Cardona, Phys. Rev. B32, 924-9 (1985); Phys. Rev. B32, 930-3 (1985).

[3] G. E. Brown, Unified Theory of Nuclear Models and Forces, 3rd Ed. (North-Holland, Amsterdam, 1971) p. 42.

[4] L. Tonks and I. Langmuir, Phys. Rev. 33, 195-210 (1929).

[5] G. Ruthemann, Ann. Physik 2, 113-34 (1948).

[6] W. Lang, Optik 3, 233-246 (1948).

[7] J. C. Slater, Insulators, Semiconductors, and Metals (McGraw Hill, New York, 1967) pp.119-125.

[8] D. Pines, Rev. Mod. Phys. 28, 184 (1956).

[9] E. D. Isaacs and P. Platzman, Physics Today, February 1996, pp.40-45.

[10] A. vom Felde, J. Sprösser-Prou, and J. Fink, Phys. Rev. B40, 10181 (1989).

[11] P. Nozières, Theory of Interacting Fermi Systems (W. A. Benjamin, New York, 1964). 